\title{
A fault identification method for marine auxiliary machinery
}

\author{
Tang Fang ${ }^{1, \mathrm{a}}$, Li Pan ${ }^{1, \mathrm{~b}}$, Xie Jianghui ${ }^{1, \mathrm{c}}$, Li Peixin ${ }^{2, \mathrm{~d}}$ \\ ${ }^{1}$ Wuhan Second Ship Design and Research Institute, Wuhan, 430205, China \\ ${ }^{2}$ HainanUniversity, Haikou, Hainan, 570228, China
}

\begin{abstract}
A large number of auxiliary equipment is set up on the shipping system, most of them is rotating machinery and in working state for most of the time, it is very important to identify health condition of equipment to find early damage and avoid fatal failure. Acoustic emission is based on rapid release of energy in case of material deformation or cracking. Auxiliary equipment will continue to emit acoustic signals, which is collected by means of a protable detection device in this paper, parameters like RMS, Peak Factor are calculated to identify health condition in time domain. Through laboratory simulation test and real ship test, the result shows that by comparing the parameters of acoustic emission signal, health condition or its change trend can be obtained, early failure of equipment can be prevented.
\end{abstract}

\section{Introduction}

In order to guarantee normal running states of ship internal systems, auxiliary machinery is set, including reciprocating pump, rotary pump, centrifugal pump, piston air compressor, refrigeration units, most of these auxiliary equipment is running all the time and belong to rotating machinery, providing the needed fresh water, sea water, air source, oil source and so on for other internal system users, once a failure occurs, it may directly affect other system working, or even threaten ship safety if it is not repaired immediately. Therefore, it is very important to carry out regular inspection and rapid fault identification of auxiliary equipment health status for whole ship normal operation. In view of marine auxiliary machinery characteristics, this paper has presented a method of acoustic emission testing for state detection, through mechanism analysis and test, analyzed the feasibility of acoustic emission testing method.

\section{Marine auxiliary machinery and fault feature}

Marine auxiliary machinery is power machinery in the ship except main engine, which can be divided into eight types: marine pumps, gas delivery machinery, deck machinery, auxiliary boiler, oil purification device, pollution prevention device, seawater desalination device, refrigeration and air conditioning device [1].

Reciprocating pumps, rotary pumps, centrifugal pumps, piston air compressors, refrigeration units and other rotating machinery is the widest wide application and the largest number in the ship, which is motor drive to transport fresh water, seawater, oil, air and other media to every user. After long time operation, internal bearing or gear of these auxiliary rotating equipment may occur wear, common problems affecting the health of rotating machinery are mainly include surface damage, lubrication failure and uniform wear[2]:

(1) Surface damage. It is usually determined by material properties, working time and impact loading strength. Long hours heavy load rotation would cause mechanical surface defects, such as pitting, peeling, etc. Due to the contact between uneven damage surface and other components, the stress point at damage location would produce an impact with the same rotating frequency, resulting in an increase in vibration and stress concentration.

(2) Lubrication failure. Due to mechanical components surface wearing, the fall of metal particles may slip into lubricant, which makes lubricant can not lubricate effectively, these particles in the gap between moving components may move irregularly with collision and extrusion of mechanical components, deform surface and make stress changes.

(3) Uniform wear. With increase of running time, wear degree of each moving mechanical component in machine increases gradually, space between each moving component becomes larger and looseness, which would produces strong continuous impact in the process of starting, reversing and running of machine.

It can be seen that in case of above early defects, if no immediate maintenance is carried out, the equipment may suffer from accelerated wear or bad operation state, such as increased vibration, abnormal sound, etc., which may lead equipment failure and shutdown eventually. Therefore, if ship can quickly determine health of auxiliary equipment, find early faults and equipment loss can be reduced.

\footnotetext{
atangfang008@163.com, ${ }^{\mathrm{b}} 497442961 @ q q . c o m$

cxjhwj666@163.com, dbeyondlpx@163.com
} 


\section{Acoustic emission detection method}

When a material deforms or cracks under force, the phenomenon of elastic waves generated by rapid release energy is called acoustic emission[3]. Acoustic emission phenomenon occurs in most materials when their shape changes or cracks. At present, acoustic emission signal frequencies generated by cracks in metal materials and some composite materials range from $10 \mathrm{kHz}$ to $1 \mathrm{MHz}[4-5]$. Based on the nature of this phenomenon, acoustic emission can be used for nondestructive detection, especially for detection of impending or initial damage in materials or structures.

Therefore, for marine auxiliary machinery, acoustic emission sensor can be used to collect signals, and equipment health state can be judged through time-domain analysis. Commonly used parameters of time-domain analysis include mean value, peak value, root mean square value, peak factor, etc.

(1) mean value, the average over signal period:

$$
\bar{X}=\frac{1}{n} \sum_{i=1}^{n} x_{i}
$$
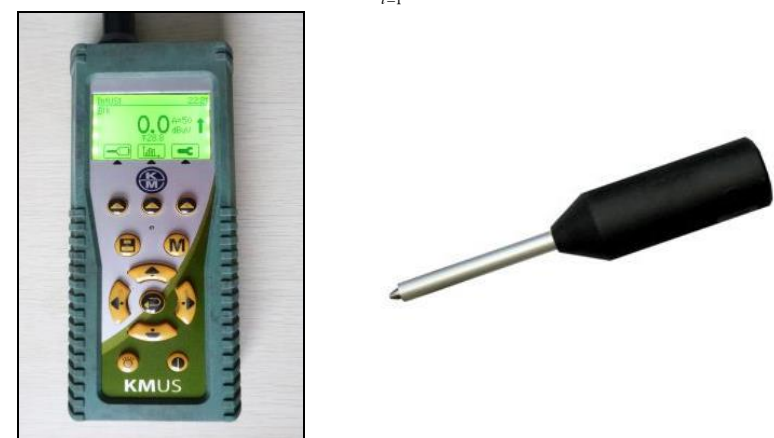

Fig.1. hand-held detection device of KMPDM

According to the structural characteristics of different ship auxiliary machinery, resonant sensor is set stably at typical parts to measure, such as motor bearing and pump shaft seal, as shown in Figure 2. The multi-function flaw detector can collect and store acoustic emission signal, after detection the acquired signal can be deduced by special software on computer for data analysis to computer indicatiors like RMS, Peak, PF, health status can be determined by comparison of the same type of equipment or changing trend.

\section{Examples of detection}

4.1 Laboratory simulation test. Three different status of motor bearings are collected in laboratory, include no significant anomaly (No.1), a slight defect in the outer ring (No.2), an obvious defect in the outer ring (No.3), as shown in Figure 3. Then they are install to rotate on the motor, the acoustic emission defection described in this article is used to test three bearings, indicatiors RMS, Peak and PF are shown in Table 1.
(2) peak value (Peak), find $\mathrm{n}$ peaks $\left\{X_{p j}\right\}$ from $\mathrm{N}$ known $x_{i}$, the Peak is:

$$
\text { Peak }=\frac{1}{n} \sum_{j=1}^{n} x_{p j}
$$

(3) root mean square value (RMS) is:

$$
R M S=\sqrt{\frac{1}{n} \sum_{i=1}^{n} x_{i}^{2}}
$$

(4) peak factor $(\mathrm{PF})$ is:

$$
P F=\frac{P e a k}{R M S}
$$

Where, $x_{i}$ is amplitude of discrete signal, $n$ is number of sampling points.

In this paper, the hand-held detection device of KMPDM company [6] is used to conduct acoustic emission detection for marine auxiliary machinery, and health status of equipment is determined according to typical parameters. The device mainly included multi-function flaw detector and resonance sensor, as shown in Figure 1.

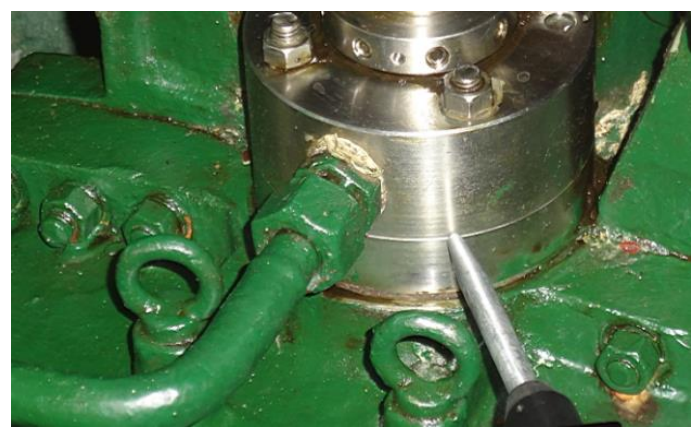

Fig.2. acoustic emission detection of marine auxiliary machinery

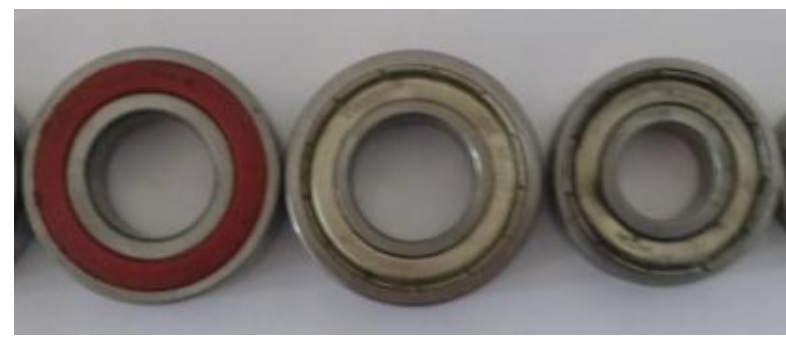

Fig.3. Bearings used in laboratory test

Table.1. Acoustic emission defection indicators of three bearings

\begin{tabular}{|c|c|c|c|}
\hline Bearing No. & RMS & Peak & PF \\
\hline 1 & 59.0 & 72.5 & 4.7 \\
\hline 2 & 59.8 & 78.2 & 8.3 \\
\hline 3 & 84.6 & 107.5 & 13.9 \\
\hline
\end{tabular}

From appearance of three bearings, bearing No. 1 is the best and bearing No. 3 is the worst, so it can be inferred that bearing No. 3 has the biggest impact during operation. From test result, it can be seen that all indicators of bearing No.1 is the smallest, while all indicators of bearing No.3 is the largest. The acoustic emission de- 
fection may be a possible method to obtain RMS, Peak, $\mathrm{PF}$ and to compare and identify health state of equipment.

4.2 Comparison test of similar equipment. There are three same centrifugal pumps in the front and rear engine cabin of a ship, one of which has a large running vibration. The method in this paper is used to collect acoustic emission data from three characteristic points(motor bearing, pump bearing and pump shaft seal) of these three pumps. According to test data, the indicators RMS of three pumps are calculated and shown in Table 2.

Table.2. RMS data of three pumps

\begin{tabular}{|c|c|c|c|}
\hline $\begin{array}{c}\text { Pump } \\
\text { No. }\end{array}$ & $\begin{array}{c}\text { motor } \\
\text { bearing }\end{array}$ & $\begin{array}{c}\text { pump } \\
\text { bearing }\end{array}$ & $\begin{array}{c}\text { pump shaft } \\
\text { seal }\end{array}$ \\
\hline 1 & 269 & 149 & 312 \\
\hline 2 & 52 & 106 & 86 \\
\hline 3 & 69 & 8 & 68 \\
\hline
\end{tabular}

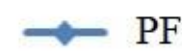

According to RMS comparison of characteristic points from three pumps, RMS value of No.3 pump is the smallest which indicates that health state is the best. RMS value of No.1 pump is the largest which indicates that health state is poor. After further inspection of No.1 pump, its bearing is nearly damaged which needs to be replaced immediately.

4.3 Compare test of the same equipment. During a period of one year, three tests are carried out for a same water pump. According to obtained acoustic emission time-domain signals every time to calculate parameters RMS, Peak, PF, changing trend curves are respected in Figure 4.

It can be seen from above trend that parameters RMS, Peak, PF have totally increased after continuous operation, vibration impact generated by pump operation have gradually increased, thus, health state gradually deteriorates, and pump is broken shortly after the third test.

\section{RMS - Peak}

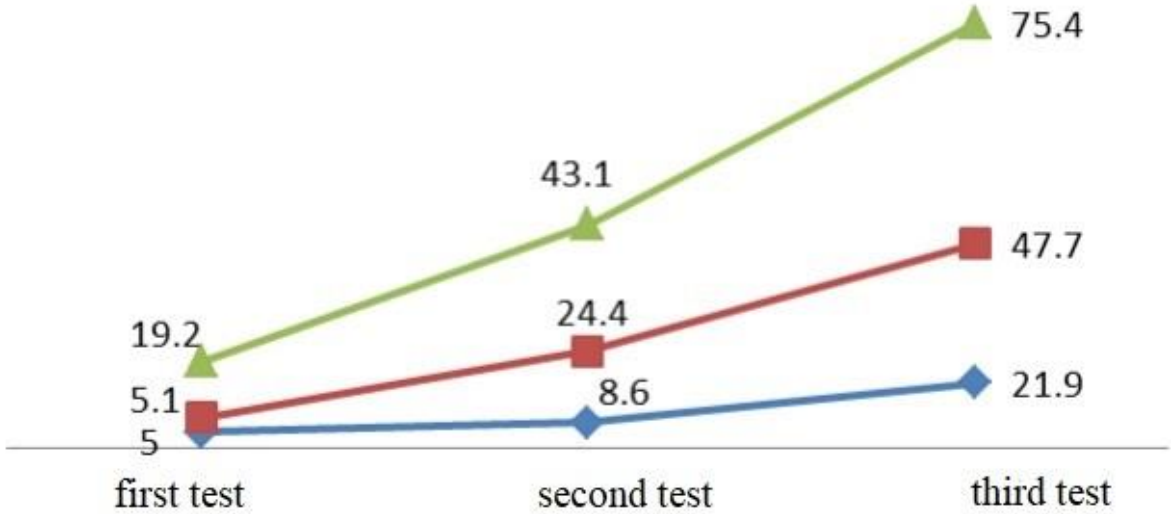

Fig.4. Trend of test results for 3 times

\section{Conclusion}

This paper has mainly analyzed main characteristics and fault types that affect health state of marine auxiliary machinery, indicated necessity of health state by quick judgement. In view of typical fault parts of auxiliary equipment, hand-held acoustic emission detection device is adopted to collect time-domain parameters of acoustic emission signal, and health state is judged according to parameters such as RMS, Peak, PF. For one marine auxiliary machinery, the same parameter value can be compared through multiple equipment of the same type in the ship, the higher value is, the poorer its health state is. Multiple tests can be conducted in a period of time to obtain the trend of changes in health parameters, database of this auxiliary equipment can be established to prevent fatal failure and reduce loss of economy.

\section{References}

1. Fu Guigen, Fei Qian. Marine auxiliary machinery. Dalian: Dalian maritime university press. 2010.

2. $\mathrm{Xu}$ Longfei. Research on fault identification of RV reducer based on acoustic emission. Kunming university of science and technology. 2015

3. J.C.Spanner. Acoustic Emission Techniques and Applications. Illiuois: Intex Puh, Co.. 1974.

4. Yang Fei. Application of acoustic emission technology in bearing fault diagnosis. Shenyang university of technology. 2009.

5. Zhao Nianwei. Gear fault monitoring based on acoustic emission technology. Shenyang university of technology. 2013.

6. KMPDM. KMPDM detector user manual. 2013. 